\title{
Numerical experimentations for a new set of local indices of a water network
}

\author{
Marco A. Bonora ${ }^{1}$, Fabio Caldarola ${ }^{1}$, Joao Muranho ${ }^{2}$, \\ Joaquim Sousa ${ }^{3}$, Mario Maiolo ${ }^{1}$ \\ ${ }^{1}$ University of Calabria, Arcavacata di Rende (CS), Italy \\ ${ }^{2}$ Universidade da Beira Interior, Covilhã, Portugal \\ ${ }^{3}$ Polytechnic Institute of Coimbra,Coimbra, Portugal \\ marcoamos.bonora@unical.it, caldarola@mat.unical.it, jmuranho@ubi.pt, \\ jjoseng@isec.pt, mario.maiolo@unical.it
}

Keywords. Urban water supply systems; performance indices; network analysis.

Very recently in CaMa, a new set of local indices has been proposed for an urban water distribution network together with a useful mathematical framework that organizes and provides the tools to treat the complex of these local parameters varying from node to node. In this talk, the applicability of such indices to various models of both abstract and real hydraulic networks will be explored through direct numerical computations and hydraulic simulations. Furthermore, starting from explicit data sets for these local parameters and using the tools offered by the mentioned mathematical setting, some well known and very frequently used global energetic indicators will be calculated in a new way and through new formulas (for example, measures of resilience, pressure, deficiency, etc.).

Finally, since the tools and the framework proposed in CaMa have the important advantage of favoring in many cases a more in-depth structural analysis of global indices, new interpretations and new implications will be briefly discussed.

\section{Acknowledgements.}

This research is partially supported by POR Calabria FESR-FSE 2014-2020, research project "origAMI, Original Advanced Metering Infrastructure" - CUP J48C17000170006.

\section{References}

[1] Caldarola F., Maiolo M. (2019) Local indices within a mathematical framework for urban water distribution systems. Submitted for publication.

[2] Muranho J., Sousa J., Sá Marques A., Gomes R. (2018) Water distribution network reliability: are surrogate measures reliable? In Proceedings of the 13th International Conference on Hydroinformatics, HIC 2018 Vol. 3 (ed. by La Loggia G. et al.), Palermo (Italy), pp. 1470-1477. 